\title{
CONCESION DE CREDITOS
}

\section{Jorge Cárdenas B.}

A

nos atrás los negocios solian exhibir una graciosa figura que oponía dos destinos. Un hombre gordo, elegantemente vestido mostraba ufano una leyenda: "Yo vendí al contado". A su costado, un hombre flaco, mal vestido y consternado se lamentaba: "Yo vendi al crédito".

Indudablemente los tiempos han cambiado, la gordura ya no es deseable y el crédito ocupa un lugar preponderante en las transacciones comerciales de hoy en dia, tanto que las tarjetas de crédito son "dinero plástico" en la mayoría de países.

Conviene reparar en que la palabra credito provienc del latín creditum, de credere, cuyo significado es creer, tener fc. Por su parte, el Diecionario de la Len. gua de la Real Academia Espanola menciona, entre otras, las siguientes acepeiones de esta palabra: derecho que uno tiene a recibir de otro alguna cosa, por lo común đinero: reputación, fama, autoridad: situación económica o condiciones morales que facultan a una persona o entidad para obiener de otra fondos o mercancias: 0 pinión que goza una persona que cum. plirá puntualmente los compromisos que contraiga.

\section{Cuándo conviene otorgar crédito}

Para los comerciantes dar credito puede ser laúnica forma de incrementar sus ven. tas, pero para las instituciones financieras es su razón de ser.

En la próctica, la política de ventas "sólo al contado" funciona para los supermercados y algunos pequeños negocios por la difusión de las tarjetas de crédito, en las que el riesgo queda cubierto por el banco o la entidad que las emile. El problema es para el resto de comerciantes y para las instituciones financic- 
ras que deben asumir en riesgo de no poder hacer efecivo el pago diniso de un tiempo prudencial y previamente acordado.

Se ha dicho tanto sobre las upligaciones de los bancos con sus depositantes, con sus clientes, con la comunidad y con el público en general, que suele olvidarse que los bancos son negocios privados, propiedad de accionistas cuyo objetivo cs obtener utilidades. Es cierto que la mayoría de los bancos operan en función de la rentabilidad a largo plazo y no para obtener una rentabilidad inmediata, y por ello identifican ese largo plazo con la prosperidad de sus clientes y la salud económica de sus comunidades; pero a pesar de ello, la banca es un negocio y se comporta como tal.

Tanto los comerciantes de bienes como los comerciantes de dinero adoptan determinados procedimientos, que difieren según el caso, para decidir el otorgamiento de los préstamos. El objetivo de este trabajo es presentar una metodología genérica para contribuir al perfeccionamiento de este procedimiento $\mathrm{y}$, al mismo tiempo, para orientar a los posibles clientes y propiciar un diálogo saludable e inteligible entre las partes.

Los procedimientos seguidos para la concesión de créditos responden a diversos criterios de acuerdo a las políticas adoptadas por cada institución. De cualquier manera, todos toman en cuenta las famosas cuatro "C" (ces) : carácter, capacidad, capital y condiciones.

La Figura 1 muestra la secuencia normal de las diversas etapas de este proceso: pero es conveniente revisar qué implica cada una de ellas.

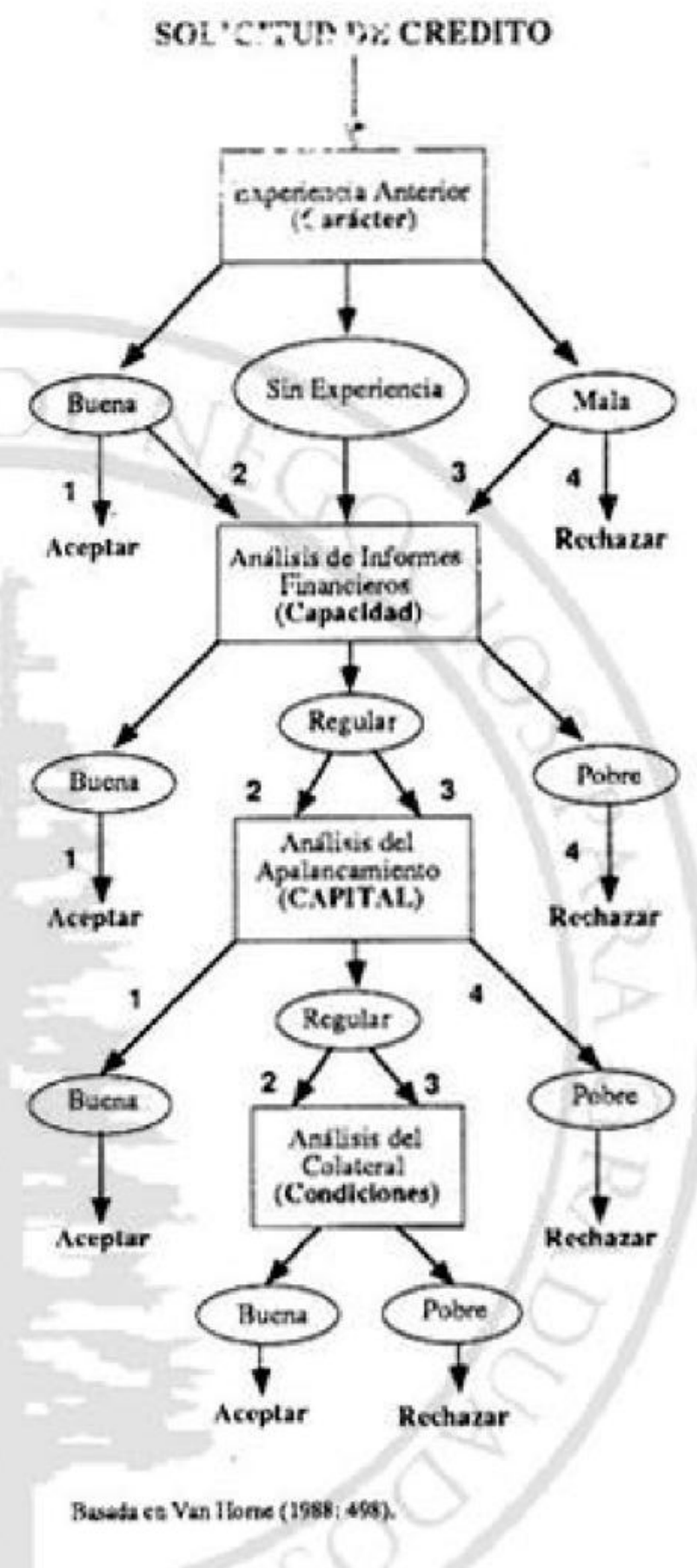

Figura 1

El carácter

El carácter es tal vez el aspecto más importante. Se refiere a la honradez de la cmpresa y de sus directivos. Expresa fundamentalmente la disposición a pagar del cliente.

La información (incluidas las expcriencias anteriores) que el banco o la entidad linanciera reúna de este aspecto es 
determinante. La dudosa "integridad" de los principales ejecutivos es crucial y o casiona normalmente ef rechazo de la solicitud del crédito, porque las consideraciones de tiempo y costo limitan la posibilidad de una extensa y escrupuiosa investigación y porque cabe la posibilidad que un "pícaro listo" sea capaz de presentar una situación aparentemente favorable.

Cuando la entidad conoce al cliente y éstc posee sanos hábitos de pago, es decir, con él no es preciso cmplear procedimientos coactivos para cl cobro, la operación dentro de los límites normales puede ser fácilmente aprobada.

De acuerdo con este criterio es conveniente que la empresa clasifique a sus clientes -y mantenga un registro de elloscgún sus hábitos de pago, en las siguientes categorías:

1. Buen pagador.

2. Paga con retraso pero la cobranza no demanda mucho esfuerzo.

3. Paga con retraso y la cobranza exige tiempo y esfuerzo considerables.

4. Incobrable o a prueba de procedimientos judiciales.

Obviamente la categoría 1 corresponde a un sujeto de credito, es fácil crcer en él y su caso no exige mayor análisis. De la misma manera, el caso opuesto, la categoría 4 , no requiere atención, está descalificada de antemano. El problema para la entidad financiera reside en los clientes de las categorias 2 y 3 . En estos casos cl otorgamiento de crédito requiere de mayores análisis y no puede ser delcgado a niveles bajos de la organización.

\section{La capacidad}

La capacidad consiste en la experiencia de la empresa en generar fondos operativamente y en la posibilidad de seguirlos generando.

Esta capacidad se puede apreciar en el movimiento histórico de fondos -en términos de caja- realizado a partir de los estados financieros ajustados por la inflación y auditados por una firma de auditores externos conforme a la norma internacional de contabilidad $N^{\circ} 7$ : Estados de Cambios en la Situación Financiera. En la Figura 2 puede observarse el resultado de este tipo de análisis. Una figura mucstra $\mathrm{cl}$ origen de los fondos, los generados por operaciones y los obtenidos por financiamiento $(\mathrm{D} / \mathrm{C})$; $y$ la $\mathrm{o}$ tra, el destino de los fondos, inversiones y la variación neta de caja.

Adicionalmente, durante la vigencia del crédito se analiza el presupuesto de caja para evaluar si la tendencia histórica se mantienc o si los cambios que surgen de las proyecciones tienen sustento.

\section{EXTRADAS POR:}

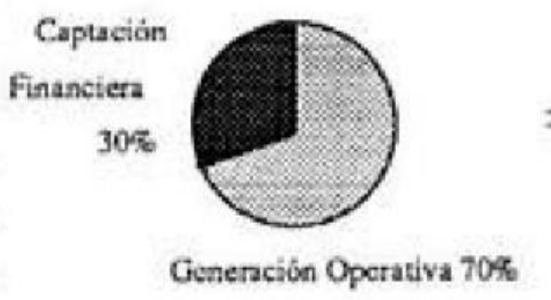

SALIDAS POR:

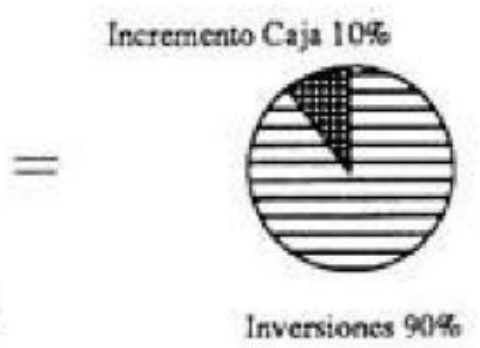


El análisis se complementa con cl examen de algunos ratios, tanto de las cifras históricas como de las proyectadas, para verificar las consistencias. Un resumen de los principales ratios se muestra en la Figura 3.

Por último, un detalle que a veces se pasa por alto es el análisis de sensibilidad que es aconsejable aplicar a las proyecciones, en especial al presupuesto de caja. Este análisis trata de encontrar las circunstancias bajo las cuales los planes previstos no producirían el efecto deseado.
Bajo el título de Modelación Financiera, la Figura 4 presenta la secuencia de los análisis descritos, que permiten clasificar las solicitudes de crédito en cuatro categorias:

\section{Alta capacidad de pago}

2. Buena capacidad

3. Regular capacidad

4. Pobre capacidad de pago

Es obvio que cl cliente con alta capacidad de pago puede ser sujeto de crédito y que el cliente con pobre capacidad de pago deberá ver rechazada su solicitud. Tam-

\section{PRINCIPALES RATIOS}

LIQUIDEZ

\section{Corriente}

Activos Corrientes

Pasivos Comentes

Acida

Activos Corrientes - Invenuarios Pasivos Comientes

\section{ACTIVIDAD}

Rotación Inventarios

$$
\frac{\text { Costo de Bienes Vendidos }}{\text { Inventarno Promedio }}
$$

Periodo Promedio Cobros

$$
\frac{\text { Cuentas promedio por cobrar }}{\text { Ventas a crédito promedio por día }}
$$

Rotación Activos

$$
\frac{\text { Ventas }}{\text { Total de Activos }}
$$

RENTABILIDAD
Margen bruto

Ventas - Costo mercadería vendida Ventas

Citilidad neta/ventas

$$
\frac{\text { Utilidad Neta }}{\text { Ventas }}
$$

Retorno patrimonio

$$
\text { Utilidad Neta }
$$

\section{APALANCAMIENTO}

Deuda/Capital

$$
\frac{\text { Deada Toual }}{\text { Patrinonio }}
$$

Interts Ganado

Utilidad antes impuestos + interés

$$
\text { Cargos por interés }
$$

Cohertura Cargos Fijos

Ingreso disponible para cubrir cargos fijos Cargos fijos 
bién aquí el banco o la cntidad financiera deberá concentrar su atención y someter a análisis adicionales a los clientes de los grupos 2 y 3 .

\section{El capital}

El capital consiste en el grado de apalancamiento financiero que mantiene la empresa, es decir, la rclación entre fondos propios y fondos ajenos, definida como estructura de capital.

La idea de palanca proviene de la física. Consiste en apoyarse en algo para que un pequeño esfuerzo $\mathrm{cn}$ una dirección dada se traduzca en un incremento mayor que el proporcional en los resultados. Análogamente, el apalancamiento financiero consiste en apoyarse en el uso de recursos ajenos para aumentar el rendimiento de los propios.

En la posición extrema, una empresa alcanzaría la máxima rentabilidad posible si no tuviera inversión propia sino exclusivamente préstamos, ya que dividir cualquier nivel de utilidad entre cero daría por resultado "infinito". No habría mayor rentabilidad posible.

Obviamente, una empresa en esa situación no ofrecería garantías para el pago de sus deudas, por la sencilla razón de que si sus niveles de venta decaen, la utilidad de operaciones no alcanzaria para pagar el servicio de la deuda.

El Cuadro 1 muestra cómo cinco empresas que obtienen la misma utilidad operativa proporcionan distinta rentabilidad a sus accionistas debido a su diferente endeudamiento. En otras palabras, MODE_ACION mบANCERA

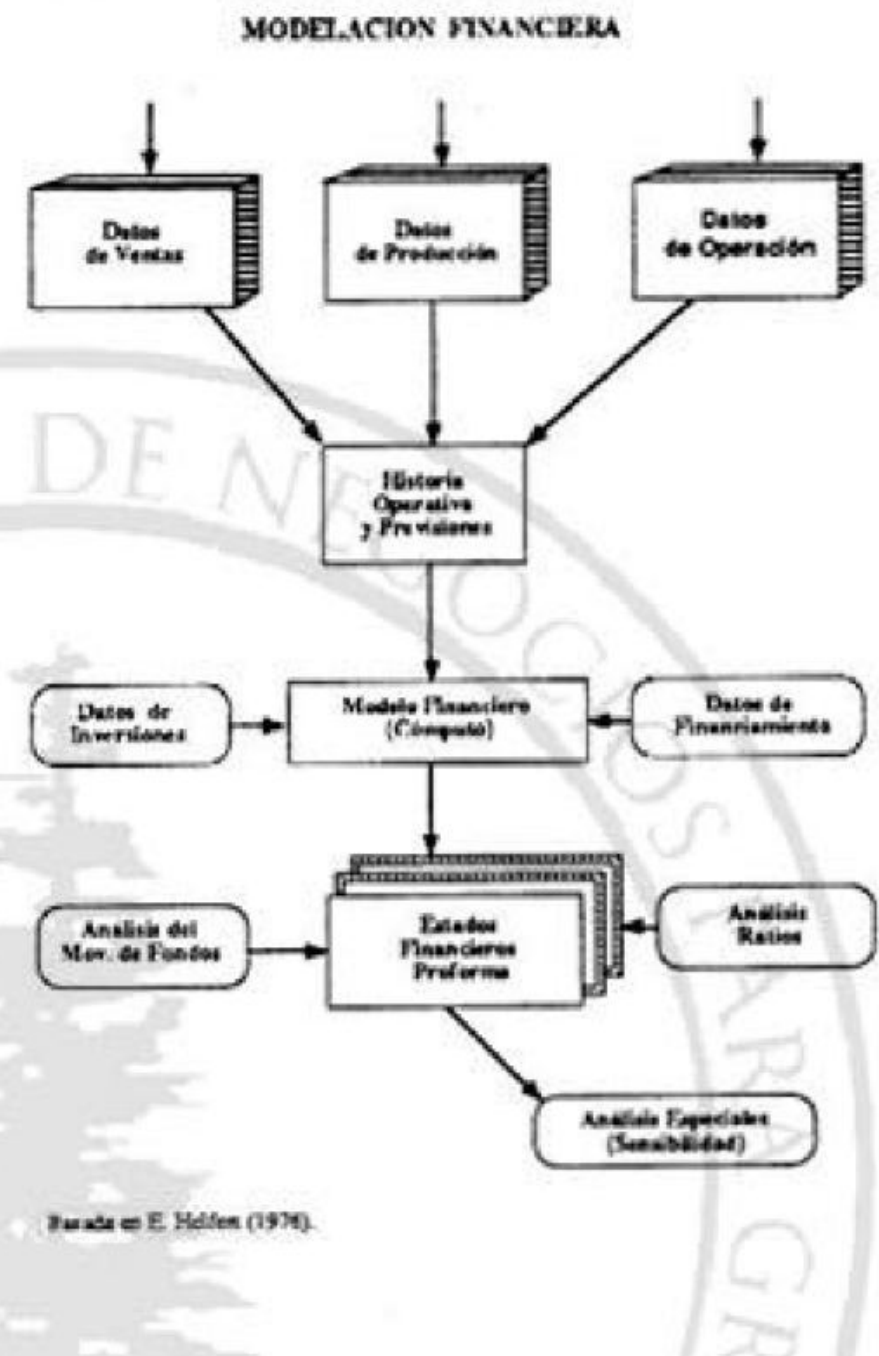

Figura 4

muestra $\mathrm{cl}$ cfocto del apalancamiento financiero sobre la rentabilidad de los accionistas.

Como puede observarse, las cinco empresas del ejemplo -A, B, C, D, Etienen las mismas características pero difieren en su estructura de capital. Cuando estas empresas obtienen una rentabilidad opcrativa (utilidades antes de impuestos $\mathrm{e}$ intereses, U.a.I.I.) alta, esto es, 44,00 en el ejemplo, los rendimientos sobre la inversión (R.O.I.) son también elevados, y los beneficios son mayores mientras mayor es el endeudamiento de la empresa. Obsérvesc cómo el R.O.I. de la empresa E, totalmente endeudada, llega hasta el infinito. 
Cuadro 1
APALANCAMIENTO FISANCIERO

\begin{tabular}{|llllll|}
\hline Caracteristicas & \multicolumn{1}{c}{ A } & \multicolumn{5}{c}{ Empresis } & B & C & D & E \\
\hline Deuda & 0 & 25 & 50 & 75 & 100 \\
Capital & 100 & 75 & 50 & 25 & 0 \\
Costo deuda & $22,00 \%$ & $22,00 \%$ & $22,00 \%$ & $22,00 \%$ & $22,00 \%$ \\
Tasa impositiva & $35,00 \%$ & $35,00 \%$ & $35,00 \%$ & $35,00 \%$ & $35,00 \%$ \\
\hline
\end{tabular}

\begin{tabular}{|c|c|c|c|c|c|}
\hline V.a.LL. & 44,00 & 44,00 & 44,00 & 44,00 & 44,00 \\
\hline Intereses & 0,00 & 5,50 & 11,00 & 16,50 & 22,00 \\
\hline U. antes impuestos & 44,00 & 38,50 & 33,00 & 27,50 & 22,00 \\
\hline Impuestos & 15,40 & 13,48 & 11,55 & 9,63 & 7,70 \\
\hline Utilidad neta & 28,60 & 25,03 & 21,45 & 17,88 & 14,30 \\
\hline R.O.I. & $2 x, 60 \%$ & $33,37 \%$ & $42,90 \%$ & $71,50 \%$ & infinito \\
\hline U.a.t.t. & 22,00 & 22,00 & 22,00 & 22,00 & 22,00 \\
\hline Intereses & 0,00 & 5.50 & 11,00 & 16,50 & 22,00 \\
\hline $\mathrm{U}$. antes impuestos & 22,00 & 16,50 & 11,00 & 5,50 & 00,00 \\
\hline Impuestos & 7,70 & 5,78 & 3,85 & 1,93 & 00,00 \\
\hline Utilidad neta & 14,30 & 10,73 & 7,15 & 3,58 & 00,00 \\
\hline R.O.1. & $14,30 \%$ & $14,30 \%$ & $14,30 \%$ & $14,30 \%$ & cers \\
\hline U.a.t.t. & 11,00 & $11, \infty 0$ & $11, \infty$ & 11,00 & 11,00 \\
\hline Intereses & 0,00 & 5,50 & 11,00 & 16,50 & 22,00 \\
\hline U. anies impuestos & 11,00 & 5,50 & 0,00 & $-5,50$ & $-11, \infty$ \\
\hline Impuestos & 3,85 & 1.93 & 0,00 & $-1,93$ & $-3,85$ \\
\hline Utilidad neta & 7,15 & 3.58 & 0,00 & $-3,58$ & $-7,15$ \\
\hline R.O.I. & $7,15 \%$ & $4,77 \%$ & $0,00 \%$ & $-14,30 \%$ & infinito \\
\hline U.a.t.I. & 5,50 & 5,50 & 5,50 & 5,50 & 5,50 \\
\hline Intereses & 0,00 & 5,50 & 11,00 & 16,50 & 22,00 \\
\hline U. antes impucstos & 5,50 & 0,00 & $-5,50$ & $-11,00$ & $-16,50$ \\
\hline Impuestos & 1,93 & 0,00 & $-1,93$ & $-3,85$ & $-5,78$ \\
\hline Utilidad neta & 3,58 & 0,00 & $-3,58$ & -7.15 & $-10,73$ \\
\hline R.o.1. & $3,58 \%$ & $0,00 \%$ & $-7,15 \%$ & $.28,60 \%$ & Infinito \\
\hline
\end{tabular}

Por el contrario, cuando la rentabilidad operativa empicza a bajar o se hace muy pequeña $(11,00$ y 5,50 en el cjemplo), la rentabilidad de los accionistas decac, y la caída es mayor micntras mayor es el endeudamiento. Obsérvese que en las empresas más endcudadas, cl R.O.I se hace cero, se vuclve negativo e incluso llega al infinito.

Sólo cuando las empresas obtienen una rentabilidad de cquilibrio $(22,00 \mathrm{cn} \mathrm{cl}$ cjemplo), los rendimientos sobre la inversión se uniformizan. En este caso no im- 
porta cual sea el nivel de endesdamicnto de las diferentes empresas, la rentivivit: dad de los accionistas será la misma. La excepción es la empresa :- tctalmente endeudads, cuyo R.O.I. se hace cero.

En función del grado de apalancamiento los clientes pueden ser clasificados, según la política de la empresa, en cuatro grupos. Nuevamente los ubicados en las catcgorias extremas no requieren mayor atención, pero el resto deberá continuar siendo analizado.

\section{Las condiciones}

Las condiciones se refieren al colateral que estará en juego, así como a la fecha prometida de pago de la deuda.

El colateral es la garantía especifica ofrecida legalmente al prestamista como respaldo de una operación de crédito. Puede estar constituida por bienes o por derechos del prestatario.

La razón para que un prestatario ofrezca en garantía sus activos es simplemente aumentar su capacidad de obtener préstamos. Para muchas empresas cuyas perspectivas son inciertas o cuyos propictarios tienen recursos limitados, otorgar garantha es su única opción para obıencr créditos, por lo que muchos banquerns preficren no aprobar un préstamo si el solicitante basa la operación en la garantía.
A pesar de lo anterior, la garantía es valiosa para el prestamista porque los rieszos de pérdidas pueden ser reducidos considerablemeri.e por.

- El contacto estrecho que se manticne con el cliente.

- El control estricto y continuo que el prestamista ejerce sobre los activos vitales del negocio, que de otra manera podrian ser entregados en garantia de otras operaciones con terceros.

- La posibilidad del prestamista, si el prestatario no puede cumplir con sus obligaciones, de incautarse la garantía y venderla para cobrar su deuda sin tener que participar en un procedimiento de quicbra.

\section{A modo de conclusión}

Tal como se ha explicado en este artículo, la concesión de créditos implica cl manejo adecuado de distintos criterios: la clección de sujetos de créditos no es tarea fácil. Sin cmbargo, quien solicita un crédito no necesariamente estú al tanto de los aspectos tenidos en cuenta por las cntidades financieras, y puede suponer, erradamente, que basta el respaldo de su capacidad financiera para obtenerlo. La secuencia presentada demuestra la importuncia de otra información; lo que al lego en la materia puede parecerle una "sutileza" es un requisito indispensable.

\section{Bibliografia Básica}

VAN HORNE, James C.. Administración financiera, sétima edición. México D.F.. Prentice Hall Hispanoamericana, 1988.

HELFERT, Erich, Técnicas de andisis finan ciero, Barcelona, Editorial Labor. 1976.
SCHALL, Lawrence y HALEY, W., Adminis. traciónfinanciera, México D.F., Mc GrawHill. 1990. 